\title{
Modeling and numerical simulations of microwave-induced ionic transport
}

\author{
S. A. Freeman a) \\ MS\&E Department, University of Wisconsin, Madison, Wisconsin 53706 \\ J. H. Booske ${ }^{\text {b) }}$ \\ ECE Department, University of Wisconsin, Madison, Wisconsin 53706 \\ R. F. Cooper \\ MS\&E Department, University of Wisconsin, Madison, Wisconsin 53706
}

(Received 24 September 1997; accepted for publication 24 February 1998)

\begin{abstract}
A numerical model was developed to simulate and study microwave-induced transport in ionic solids. The model is based on continuum equations, is very general, and could be applied to many materials. The assumptions, boundary conditions, initial conditions, and numerical techniques used in the model are described. Results are presented from a study of microwave driven defect transport in sodium chloride. Static, high-frequency, and quasistatic results show that ponderomotive rectification of vacancy fluxes will act to deplete the vacancies in a near-surface region and will continue to pull vacancies to the surface through diffusion kinetics. The ponderomotive driving force for this transport is characterized over a wide range of variable space. The magnitude of the driving force falls right in the range such that it can explain why microwave-enhanced mass transport is observed in some experimental cases but not in others. (C) 1998 American Institute of Physics. [S0021-8979(98)02311-1]
\end{abstract}

\section{INTRODUCTION}

The heating of a material depends on two physical processes. First, energy must be absorbed and converted to heat through high-energy gas particles colliding with the surface of the material (convection heating) or via an interaction between the material and electromagnetic fields (radiation heating). Second, the absorbed heat will transfer within the material via electron or phonon energy transfer (thermal conduction). High-temperature heating of ceramic materials usually involves heating the surface of the sample with infrared radiation, but heating rates are limited by the slow rate of inward heat conduction due to the typically low thermal conductivities of ceramics. Microwave radiation, on the other hand, penetrates deeper, allowing for more volumetric heating and relying less on thermal conduction.

Microwave heating has niche applications for materials with certain absorption and thermal conduction properties, and many ceramic materials fall into this category. In recent years, many researchers have studied high-temperature microwave processing of ceramics such as firing, sintering, joining, and melting. ${ }^{1}$ The initial stimuli for their efforts were the unique benefits that microwave heating might provide that conventional heating could not. These anticipated benefits included more precise and controlled volumetric heating, faster ramp-up to temperature, lower energy consumption, and improved quality and properties of the processed material.

Besides the anticipated benefits, many experimenters have also observed an apparent "nonthermal" effect in the

\footnotetext{
a) Now at Hewlett-Packard, Microwave Technology Division, Santa Rosa, CA 95403.

${ }^{b)}$ Electronic mail: booske@engr.wisc.edu
}

form of enhanced kinetics. Specific observations in ceramic materials include enhanced rates of sintering, ${ }^{2,3}$ grain growth, ${ }^{4}$ surface-penetration diffusion, ${ }^{5,6}$ joining, ${ }^{7,8}$ and nucleation. ${ }^{9}$ There is also much anecdotal evidence for microwave enhancements of polymer processing, epoxy curing, catalysis, and reactions in organic materials.

An important recent breakthrough in the understanding of these empirical observations has been the identification of a fundamental mechanism which nonthermally enhances solid state ionic mass transport. This previously unrecognized phenomenon ${ }^{10,11}$-articulated as a "ponderomotive force" ( $(p m f)$ - is now understood to be a fundamental property of microwave-irradiated interfaces that represent abrupt discontinuities in ionic mobility. Microwave-excited ionic currents become locally rectified (near the interface), giving rise to an additional driving force for mass transport. Preliminary experimental evidence for the action of the microwave ponderomotive force was first reported, ${ }^{12}$ and subsequent confirmation was realized by experimental observation of very specific, theoretically predicted transient decay dynamics of $p m f$-induced ionic currents in halide salt crystals. ${ }^{11}$ From a mathematical standpoint, microwave ponderomotive forces at material interfaces are explicitly revealed upon nonlinear perturbation analysis of three fundamental equations applied to a material interface or surface: the continuity equation, Gauss's Law, and the mass transport equation for mobile ionic species. This is discussed in greater detail in the body of this paper. A critical point to emphasize, however, is that no additional model-specific assumptions are needed to derive the existence of the microwave $p m f$ from the three quoted equations from which it is derived; it can be active for crystalline (Schottky or Frenkel disorder) or amorphous microstructures. 
In order to more broadly explore what effect the ponderomotive force would have on various classes of masstransport processes, we developed a numerical model to simulate the defect perturbations and fluxes that result from microwave excitation of ionic crystalline solids. This paper describes the model and computer simulations used to visualize the ponderomotive effect and to calculate the resulting driving force for mass transport.

\section{MODEL DESCRIPTION}

The basis of the ponderomotive theory derives from applying perturbation techniques to a coupled set of nonlinear partial differential equations that describe mass and charge flow in conducting media in general and ionic solids in particular. The three differential equations used are continuum equations utilized to describe physical problems in plasma physics, semiconductor physics, electrochemistry, and materials science. In fact, the term "ponderomotive" is borrowed from a similar phenomenon in plasma physics. "Ponderomotive" derives from the Latin words pondus (weight or mass) and motive (to move); a ponderomotive force is thus an electromagnetic force that tends to move mass.

The first differential equation describes the flux $(J)$ of a charged species $(n)$ that results from either a concentration gradient $(\nabla C)$ or an electrical potential gradient $(\mathbf{E}=$ $-\nabla V)$ :

$$
\mathbf{J}_{n}=-D_{n} \boldsymbol{\nabla} C_{n}+\frac{f D_{n} C_{n}}{k T} q_{n} \mathbf{E},
$$

where $D$ is the diffusion coefficient, $q$ is the electric charge of the species, $k T$ is the thermal energy, and $f$ is the correlation coefficient that relates self-diffusion to ionic conduction. The correlation coefficient varies typically between 0.5 and 1 , depending on the crystalline structure and diffusion direction, so we ignored it throughout the rest of this work. It is also noted that Eq. (1) is written in terms of particle flux, not charge flux. The two terms on the right-hand side of Eq. (1) are called diffusion and drift, respectively. The diffusion term (Fick's Law) assumes ideal solution behavior, and the drift term assumes Ohmic conduction. Chemical potential gradients can be opposed and balanced by electrical potential gradients at a state of equilibrium (i.e., zero flux).

The second important equation is the continuity condition:

$$
\frac{\partial C_{n}}{\partial t}=-\boldsymbol{\nabla} \cdot \mathbf{J}_{n}+S_{n},
$$

which states that the time-rate-of-change of concentration depends on the divergence of the particle flux and the presence of sources/sinks $(S)$.

The third equation is Gauss's Law (differential form):

$$
\boldsymbol{\nabla} \cdot \mathbf{E}=\frac{1}{\varepsilon_{r} \varepsilon_{0}} \sum_{n} q_{n}\left(C_{n}-C_{n}^{i}\right),
$$

which states that nonneutral accumulations of (mono) polar charge serve as (flux) sources of electric field. In Eq. (3), $\varepsilon_{0}$ is the permittivity of free space, $\varepsilon_{\mathrm{r}}$ is the relative dielectric constant of the solid (assumed to be independent of the field strength), and the superscript $i$ denotes the initial, equilibrium value of the concentration.

In the simulation work, we desired to apply Eqs. (1)-(3) to the case of high-power microwave fields interacting with charged defect species in an ionic solid. A few key assumptions were used. First, since grain sizes are typically much smaller than microwave wavelengths, we assumed that the electric field strength was uniform across the dimension of the crystal. Second, only the normal component of the electric field was considered, so a one-dimensional model was used with the assumption that the electric field strength was aligned perpendicular to the crystal surface. Third, only half of a single crystal was considered such that one boundary was the surface, and the other edge was the bulk or interior of the crystal.

For the results described here, we simulated the ponderomotive effects in sodium chloride. We allowed for doping of $\mathrm{NaCl}$ with divalent impurities (e.g., $\mathrm{Ca}^{2+}$ ). However, the impurities were assumed to be immobile, and only $\mathrm{Na}$ and $\mathrm{Cl}$ vacancy migration was simulated. $\mathrm{NaCl}$ was chosen because of its ideal properties as an ionic solid and because it has well-characterized defect parameters (formation energies and mobilities). The generality of the effects studied here is not lost by the choice of $\mathrm{NaCl}$, however, since the continuum equations could be derived for any mobile charged species in any material. Thus, the model is very general, and the results can be expected to occur in many solid-state systems.

\section{A. Perturbations}

The equations were solved numerically with the aid of perturbation techniques. Perturbations from the initial values were allowed for the defect concentrations and the electric potential/field:

$$
\begin{aligned}
C_{n}(x, t) & =C_{n}^{i}(x)+C_{n}^{p}(x, t), \\
V(x, t) & =V^{i}(x)+V^{p}(x, t)+V^{m w}(x, t), \\
E(x, t) & =-\frac{\partial V(x, t)}{\partial x} \\
& =E^{i}(x)+E^{p}(x, t)+E^{m w}(t) .
\end{aligned}
$$

The subscript $n$ refers to the vacancy species, the superscript $i$ indicates the initial, equilibrium values (unchanging in time), the superscript $m w$ denotes the microwave potential/ field, and the superscript $p$ refers to the perturbation (varying spatially and temporally) from the initial value due to the microwave excitation.

The perturbation Eqs. (4)-(6) were then applied to the differential Eqs. (1)-(3) for the 1D case. It was illustrative to break the flux Eq. (1) into separate components. The diffusion flux term yielded two flux components $(J 1, J 2)$ :

$J 1_{n}=-D_{n} \frac{\partial C_{n}^{i}(x)}{\partial x}$ and $J 2_{n}=-D_{n} \frac{\partial C_{n}^{p}(x, t)}{\partial x}$.

The drift term yielded a total of six components, three involving $C_{n}^{i}(J 3-J 5)$ : 


$$
\begin{aligned}
& J 3_{n}=\frac{D_{n} q_{n}}{k T} C_{n}^{i}(x) E^{i}(x), \\
& J 4_{n}=\frac{D_{n} q_{n}}{k T} C_{n}^{i}(x) E^{p}(x, t), \\
& J 5_{n}=\frac{D_{n} q_{n}}{k T} C_{n}^{i}(x) E^{m w}(t),
\end{aligned}
$$

and three involving $C_{n}^{p}(J 6-J 8)$ :

$$
\begin{aligned}
& J 6_{n}=\frac{D_{n} q_{n}}{k T} C_{n}^{p}(x, t) E^{i}(x), \\
& J 7_{n}=\frac{D_{n} q_{n}}{k T} C_{n}^{p}(x, t) E^{p}(x, t), \\
& J 8_{n}=\frac{D_{n} q_{n}}{k T} C_{n}^{p}(x, t) E^{m w}(t) .
\end{aligned}
$$

The total flux for each species was obtained from a superposition of the components.

Application of the perturbations to the continuity condition yielded separate conditions for each species:

$\frac{\partial C_{\mathrm{Na}}^{p}}{\partial t}=-\frac{\partial J_{\mathrm{Na}}}{\partial x}+S_{\mathrm{Na}}$ and $\frac{\partial C_{\mathrm{Cl}}^{p}}{\partial t}=-\frac{\partial J_{\mathrm{Cl}}}{\partial x}+S_{\mathrm{Cl}}$.

In Gauss's Law, however, the $\mathrm{Na}$ and $\mathrm{Cl}$ vacancy concentrations were coupled, but the initial and perturbation values were separable into the following:

$$
\begin{aligned}
& \frac{\partial E^{i}(x)}{\partial x}=\frac{e}{\varepsilon_{0} \varepsilon_{r}}\left[C_{\mathrm{Cl}}^{i}(x)+C_{\mathrm{dop}}^{i}(x)-C_{\mathrm{Na}}^{i}(x)\right], \\
& \frac{\partial E^{p}(x, t)}{\partial x}=\frac{e}{\varepsilon_{0} \varepsilon_{r}}\left[C_{\mathrm{Cl}}^{p}(x, t)-C_{\mathrm{Na}}^{p}(x, t)\right],
\end{aligned}
$$

where the subscript $n=$ dop represents the divalent dopant. The numerical solution procedure was to simultaneously solve Eqs. (14) and (16) while making use of the flux Eqs. (7)-(13). Of course, a solution could be found only if boundary conditions and initial conditions were correctly specified.

\section{B. Boundary conditions}

The 1D model had two boundaries-the crystal surface at $x=s$ and the bulk or interior of the crystal at $x=b$. Boundary conditions were specified at each boundary for both the continuity equation and Gauss's Law.

We considered first the surface boundary conditions. For Gauss's Law the electrical potential (or one of its derivatives) must be specified at the surface. Since the reference value of the potential could be arbitrarily assigned, we chose to define the potential to be zero at the surface and use that as a boundary condition:

$$
\left.V\right|_{x=s}=0 .
$$

For the continuity equation, we recognized several physically unique features of the surface. First, in the absence of dislocations (another assumption of our simulations), vacancies are created and annihilated only at the surface of the crystal. Therefore, the source/sink term $S$ is nonzero only at the surface. Second, we recognized that at the surface, the flux of vacancies from the crystal into the neighboring region (either atmosphere or a neighboring crystal) is impeded, and there is an abrupt nonuniformity in the transport properties:

$$
\left.\frac{\partial J_{n}}{\partial x}\right|_{x=s} \neq 0 .
$$

We investigated three candidate boundary conditions for the continuity equation. In the first candidate (called the "no-sink" condition), no vacancy creation or annihilation was allowed, and the surface was considered impermeable to vacancy fluxes:

$$
\left.J_{n}\right|_{x=s}=0 \quad \text { and }\left.\quad S_{n}\right|_{x=s}=0 .
$$

In the second candidate, the source/sink term was handled very much like generation and recombination of electrons and holes in semiconductors:

$$
\left.J_{n}\right|_{x=s}=0 \quad \text { and }\left.\quad S_{n}\right|_{x=s}=\frac{-\left.C_{n}^{p}\right|_{x=s}}{\tau_{n}},
$$

$\tau$ was a time constant associated with the exchange of excess vacancies at the surface with the neighboring region. Under certain simulation conditions, however, this boundary condition leads to numerical instability. In the third candidate, similar to the second, the exchange of vacancies at the surface depended on the perturbation of their concentration. In this approach, however, the source/sink action was incorporated into the flux action:

$$
\left.J_{n}\right|_{x=s}=-D_{n} \frac{\left.C_{n}^{p}\right|_{x=s}}{d} \text { and }\left.\quad S_{n}\right|_{x=s}=0 .
$$

The second and third candidate approaches produced similar results, but the third was more numerically stable since the flux calculation depended on the diffusion jump distance $d$ (related to lattice spacing) and not the numerical mesh spacing. Later we will discuss results obtained using both Eq. (19) (no-sink) and Eq. (21) (sink) conditions.

In the bulk of the crystal, there were no sources or sinks for vacancies, so $S_{\mathrm{n}}=0$. The interior boundary was assumed to be the center point of the crystal, such that symmetry conditions applied and the divergence of the flux and electric field were zero:

$$
\left.\frac{\partial J_{n}}{\partial x}\right|_{x=b}=0 \quad \text { and }\left.\quad \frac{\partial E}{\partial x}\right|_{x=b}=0 .
$$

Boundary condition (22), applied to Eq. (14), implied that the bulk vacancy concentrations would not change over time from their initial values; this constraint will be discussed in the results.

\section{Initial conditions}

The initial conditions of the vacancy and dopant concentrations and the electrical potential were a necessary and important part of the simulations. The previous theoretical work $^{11,12}$ had only considered defect concentrations that 
were initially uniform, but we expanded the model to allow for preexisting chemical and electrical potential gradients.

The concept of space-charge layers in ionic materials has been physically and mathematically developed. ${ }^{13,14}$ For lightly doped alkali halides, the result is that cation vacancy concentrations are enhanced near the surface, anion vacancies are depleted, and a large, near-surface electric field holds the concentration gradients in check. Our simulations incorporated the model of Kliewer and Koehler to describe the initial conditions in the crystal. Initial vacancy concentrations $\left(C_{n}^{i}\right)$ were calculated using standard defect chemistry including extrinsic doping with divalent cation impurities (stress effects from doping and dipole-defect interactions were not considered). The initial electrical potential $\left(V^{i}\right)$ and electric field $\left(E^{i}\right)$ were also calculated from the model. Vacancy formation energies ${ }^{13}$ and vacancy mobility parameters ${ }^{15}$ were taken from the literature.

\section{Numerical techniques}

After the initial conditions were determined, we solved numerically Eqs. (14) and (16) simultaneously with the boundary conditions. For this purpose, we used the Simulation Generator (SIMGEN) software package. ${ }^{16,17}$ The program employed a finite-difference calculation based on the Newton method. It also included Gaussian elimination techniques and Newton damping.

Another feature we used was the Scharfetter-Gummel discretization technique, ${ }^{17,18}$ which is important when the spatial variation of the electrical potential is large over a single mesh (discretization) step. We found this approach to be critical at higher doping levels, when the inherent boundary space-charge layer achieved steep potential gradients. The characteristic distance for the space-charge layer is the Debye-Hückel (DH) distance $(\lambda)$ :

$$
\lambda_{n}=\sqrt{\frac{D_{n} \varepsilon}{2 \sigma_{n}}}=\sqrt{\frac{\varepsilon_{0} \varepsilon_{r} k T}{2 C_{n}^{i} q_{n}^{2}}}
$$

which is smaller at higher defect concentration levels.

The numerical techniques required mesh specifications for the time $(\Delta t)$ and spatial $(\Delta x)$ dimensions. The time step was chosen to be fine enough such that sinusoidal functions of the microwave field were smoothly replicated; typically $1 / 32$ of a cycle was used. The spatial mesh was then calculated and adjusted to satisfy the numerical stability criteria for diffusion: ${ }^{19}$

$$
D_{n} \leqslant \frac{\Delta x^{2}}{2 \Delta t} .
$$

\section{RESULTS AND DISCUSSION}

The validity of the numerical modeling was tested in several ways. First, we ran the simulations with a zero amplitude microwave field and observed no perturbations from the initial conditions. Second, we applied a step function in the concentration at the surface and found the resulting flux dynamics to follow diffusion behavior. Third, when no source or sinks were included in the model, we found that the total number of particles and total charge were con-
TABLE I. Adjustable variables explored in the simulations.

\begin{tabular}{lcc}
\hline \multicolumn{1}{c}{ Variable } & Range & Example case \\
\hline Microwave $E$ field & $10^{5}-10^{6}$ & $10^{6}$ \\
$\quad$ strength $(\mathrm{V} / \mathrm{m})$ & & \\
Microwave frequency $(\mathrm{Hz})$ & $10^{6}-10^{10}$ & $10^{9}$ \\
Crystal temperature $(\mathrm{K})$ & $400-1000$ & 800 \\
Crystal doping (mole fraction) & $10^{-6}-10^{-3}$ & $10^{-5}$ \\
\hline \hline
\end{tabular}

served. Because the model gave valid results for these tests, we trust it gave valid results for simulations of the ponderomotive effect during microwave field application. Adjustable variables in the model included the microwave field strength, the microwave frequency, the sample temperature, and the amount of divalent cation doping in the sample. Ranges for these variables are listed in Table I.

We classified the results of the simulations into three categories-static, high frequency (HF), and quasistatic (QS). The static results were determined solely by the initial conditions. The HF results showed the sinusoidal character of the perturbations. The QS results, obtained by time averaging the HF results over one cycle, showed the slowly varying nature of the perturbations. For clarity, we present here static, HF, and QS results all from the same simulation (the "example" values in Table I). Finally, we examined the ponderomotive force $p m f$ that we calculated from the results of our simulations, and characterized it within the variable space laid out in Table I.

\section{A. Static results}

The static results did not cause any defect fluxes, perturbations of vacancy concentrations, or perturbation of the electric field and, thus, showed only the spatial variations of the initial values. However, the static case strongly affected the HF and QS results, and so were worth some examination.

Our model of the space-charge effects at the interface yielded similar concentration profiles to those reported in Ref. 13. However, we found it more enlightening to plot the profiles on a linear scale rather than on the standard logarithmic scale, where the phenomenon appears quite symmetric. The DH screening distance is seen in Fig. 1 for the example case. The surface boundary is on the right-hand side of the plot, while the bulk of the crystal is towards the left. (Only $40 \mathrm{~nm}$ are shown, although the values were calculated to a depth of approximately $150 \mathrm{~nm}$.) We see in Fig. 1 that the distance scalings for $\mathrm{Na}$ vacancy enhancement and $\mathrm{Cl}$ vacancy depletion appear different, and this difference was also noticed in the HF and QS results discussed below. The initial electric potential $\left(V^{i}\right)$ similarly varied over the length of the $\mathrm{DH}$ distance from $0 \mathrm{~V}$ at the surface to approximately -0.2 $\mathrm{V}$ in the bulk. The initial electric field $\left(E^{i}\right)$ also varied over the $\mathrm{DH}$ distance from $0 \mathrm{~V} / \mathrm{m}$ in the bulk to approximately $-4 \times 10^{7} \mathrm{~V} / \mathrm{m}$ at the surface.

The scale length of the space charge and segregation effects merits some discussion. As the doping level was increased in the simulations, the DH distance became smaller. As mentioned above, when it becomes small enough, the electrical potential changes over a very short distance and the 

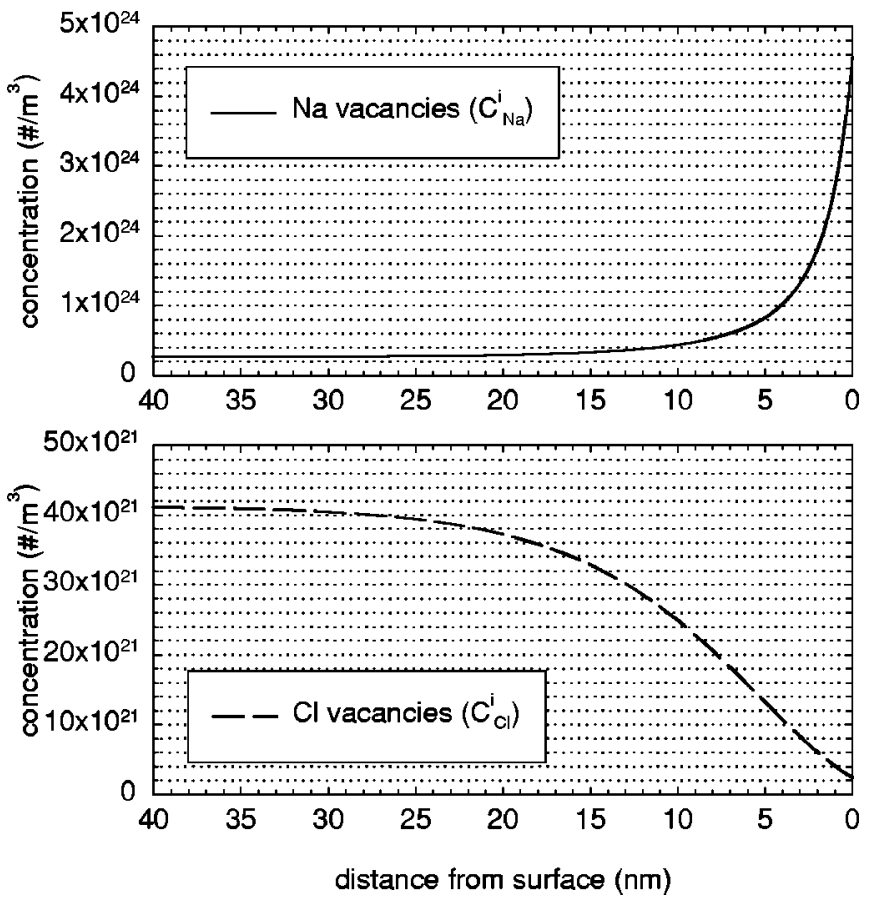

FIG. 1. The initial concentration profiles $\left(C_{n}^{i}\right)$ for $\mathrm{Cl}$ vacancies and $\mathrm{Na}$ vacancies plotted on a linear scale.

Scharfetter-Gummel discretization technique must be employed. It is also important to consider the DH length in comparison to the characteristic diffusion length $(\ell)$ during a microwave cycle of frequency $f$ :

$$
\ell_{n} \approx \sqrt{\frac{D_{n}}{f}}
$$

since $\ell$ is the distance within the crystal over which the ponderomotive action due to the surface boundary condition takes effect. For our simulations of $\mathrm{NaCl}$, the $\mathrm{DH}$ distance was typically longer than the diffusion distance for most of the variable space we probed. The two lengths become approximately equivalent when the ionic conductivity $(\sigma)$ is about the same as the dielectric conductivity $(\omega \varepsilon)$.

\section{B. High-frequency results}

To obtain a ponderomotive effect, a high-frequency excitation must cause HF vacancy perturbations. In our model, this was principally found in the $J 5$ flux term [Eq. (10)]. Whenever $J 5$ was spatially nonuniform, HF concentration perturbations occurred by virtue of Eq. (14). Gradients in the vacancy fluxes derived from two sources. One was the abrupt flux disruption at the surface boundary [Eq. (18)]. Another source was the nonuniform initial vacancy concentrations shown in Fig. 1. Since $C_{n}^{i}$ values were nonuniform, the J5 fluxes were also nonuniform.

In Fig. 2 we plot the spatial variation and time evolution of $J 5_{\mathrm{Cl}}$ for the same example presented in Fig. 1. The vertical axis shows the value of the flux. The surface of the crystal is on the forward-right side of the plot, and the interior of the crystal towards the back left. The time axis starts at $t=0$ at the back right of the plot and evolves in time towards the front left. Two full cycles of microwave fields at $1 \mathrm{GHz}$ and

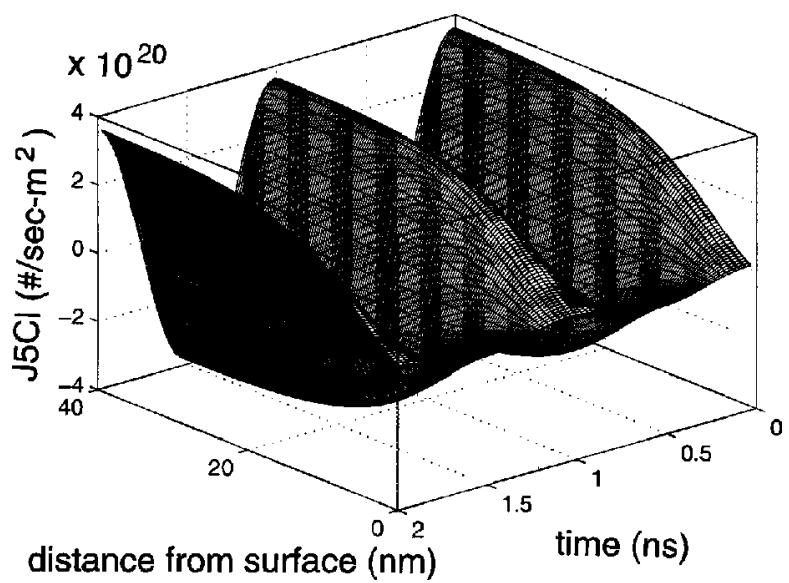

FIG. 2. The HF flux $J 5$ of $\mathrm{Cl}$ vacancies near the surface boundary.

$10^{6} \mathrm{~V} / \mathrm{m}$ are shown. The flux decreases as it approaches the surface because the initial concentration of $\mathrm{Cl}$ vacancies is depleted within the DH layer [see Fig. (1)]. Not seen here is the very abrupt flux nonuniformity at the boundary because we are only plotting $J 5$ and not the total flux.

The $J 5$ fluxes were exactly sinusoidal in time, so no net flux was derived by time averaging the $J 5$ term of the flux. Yet, because of the nonuniformity of $J 5$, vacancy concentrations were perturbed from their initial values, as per Eq. (14). The $\mathrm{Cl}$ vacancy perturbations $\left(C_{\mathrm{Cl}}^{p}\right)$ that resulted from the spatial nonuniformity of $J 5_{\mathrm{Cl}}$ are shown in Fig. 3. The two distance scales $-\ell$ and $\lambda$-are clearly seen here. The diffusion effect was localized within a few nanometers of the surface, while the DH scale extended over more than the 20 $\mathrm{nm}$ distance plotted here. Diffusion occurred because of the $J 2$ flux, so that when the concentration was perturbed abruptly at the surface, some vacancies would diffuse back into the crystal over a length characterized by $\ell$.

The HF Na vacancy perturbations $\left(C_{\mathrm{Na}}^{p}\right)$ are plotted in Fig. 4. The perturbations within 1-2 $\mathrm{nm}$ of the surface are most apparent, indicating that for $\mathrm{Na}$ vacancies, the surface boundary condition produced the most ponderomotive action. The effect of the DH screening layer is difficult to see in Fig. 4, but it was the cause of the subsurface $(3+\mathrm{nm})$ vacancy concentration oscillations that were $180^{\circ}$ out of

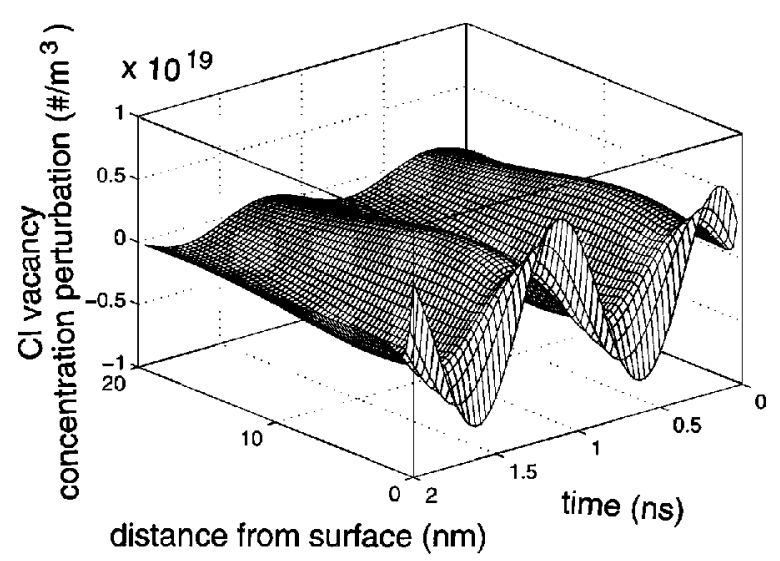

FIG. 3. The HF perturbations of $\mathrm{Cl}$ vacancy concentration $\left(C_{\mathrm{Cl}}^{p}\right)$ near the surface boundary. 


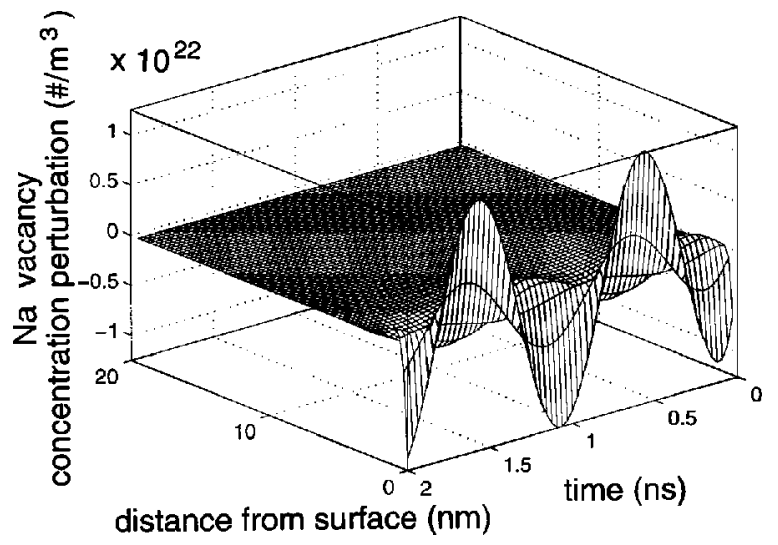

FIG. 4. The HF perturbations of Na vacancy concentration $\left(C_{\mathrm{Na}}^{p}\right)$ near the surface boundary.

phase with respect to the surface level oscillations. In the case of $\mathrm{Cl}$ vacancies, the space-charge effects and the surface boundary condition both acted to retard the $\mathrm{J}_{\mathrm{Cl}}$ flux. Thus, both the effects acted together and were in phase. On the other hand, the enhanced $\mathrm{Na}$ vacancy concentration within the DH layer acted to accelerate the J5 flux, while the boundary condition retarded the flux. One therefore sees the two effects in Fig. 4 to be out of phase.

Because the oppositely charged $\mathrm{HF} \mathrm{Na}$ and $\mathrm{Cl}$ vacancy perturbations were (1) of different magnitude and (2) $180^{\circ}$ out of phase with each other, there existed a HF electric field perturbation, as follows from Eq. (16). The perturbation field $\left(E^{p}\right)$ is plotted for the same two microwave cycles in Fig. 5. It was $180^{\circ}$ out of phase with respect to the microwave field, so it acted in opposition to the microwaves. However, the magnitude of $E^{p}$ was usually only a few percent (2\% in this case) of $E^{m w}$.

To this point we have shown how the initial conditions and the boundary conditions lead to spatially nonuniform fluxes and HF concentration oscillations. The stage is thus set for ponderomotive action, since the HF concentration perturbations are multiplied by the sinusoidal driving fields in Eqs. (12) and (13). The $J 7$ and $J 8$ fluxes had a strong sine $^{2}$ nature to them. This is clearly seen in Fig. 6 for $J 8_{\mathrm{Cl}}$.

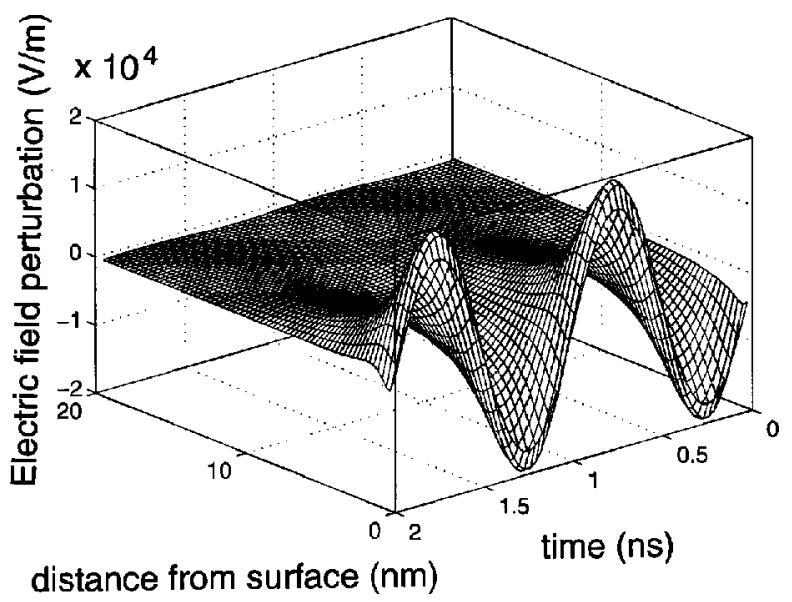

FIG. 5. The HF perturbations of the electric field $\left(E^{p}\right)$ near the surface boundary.

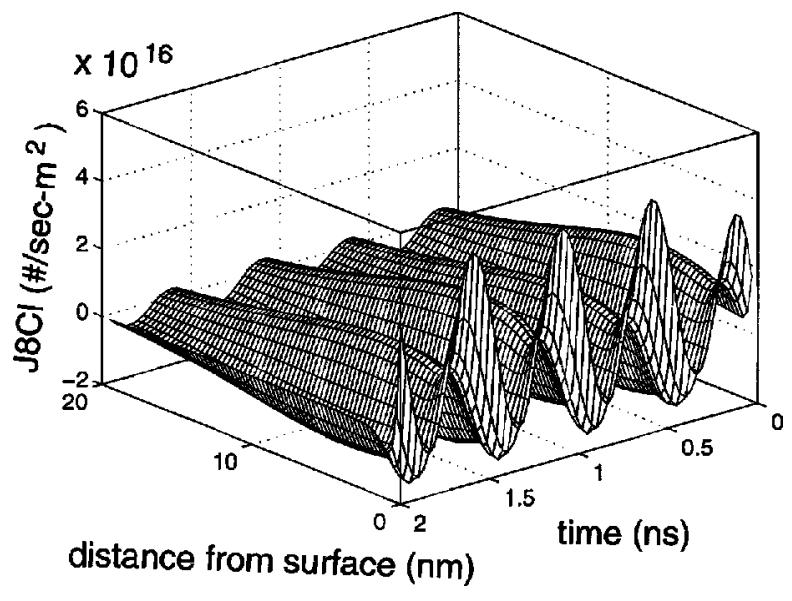

FIG. 6. The $J 8_{\mathrm{Cl}}$ flux showed sine $\mathrm{e}^{2}$ behavior near the surface.

Again, two microwave cycles are being plotted, but this time we observed four peaks instead of two, as is indicative of a $\operatorname{sine}^{2}$ function. Some of the values were in fact negative because there was a slight phase shift (due to the diffusion time delay) between the application of the sinusoidal microwave field and the resulting sinusoidal concentration perturbations. Plots of $J 7_{\mathrm{Cl}}, J 7_{\mathrm{Na}}$, and $J 8_{\mathrm{Na}}$ all showed similar sine ${ }^{2}$ behavior.

The ponderomotive action (seen in Fig. 6) caused rectification of vacancy fluxes near the surface. As we will show in the following section, this effect produced a time-averaged nonzero (quasistationary) flux of particles within the crystal. We will next examine how the high-frequency rectification acted as a driving force for mass transport.

\section{Quasistatic results}

The results we have presented so far are illustrative of the common qualitative phenomenology observed in most of the simulations. Of course, the values of temperature and doping impacted the specific magnitude of the DH screening length near the surface boundary. The magnitude of the HF perturbations also depended on the field strength, and the diffusion scaling $\ell$ depended on the frequency. Nevertheless, the HF results were qualitatively similar over a wide range of variable space. They were also somewhat insensitive to the exact form of the surface boundary condition. The same cannot be said, however, about the quasistatic results. They were obtained by time averaging the HF effects and therefore measured the amount of asymmetry or nonsinusoidal nature of the HF oscillations. The magnitude of the QS results were therefore much smaller than the HF resultsusually by several orders of magnitude. The QS results also varied from case to case. In fact, the case study we are presenting here gave slightly atypical QS results. We will therefore complement it with QS results from another set of simulation variables in order to show the more typical results and to give the reader some sense of the complex nature of this phenomenon.

The results we present here all derived from simulations with boundary condition (21), so we will first discuss the effect of the surface boundary condition on the QS results. 


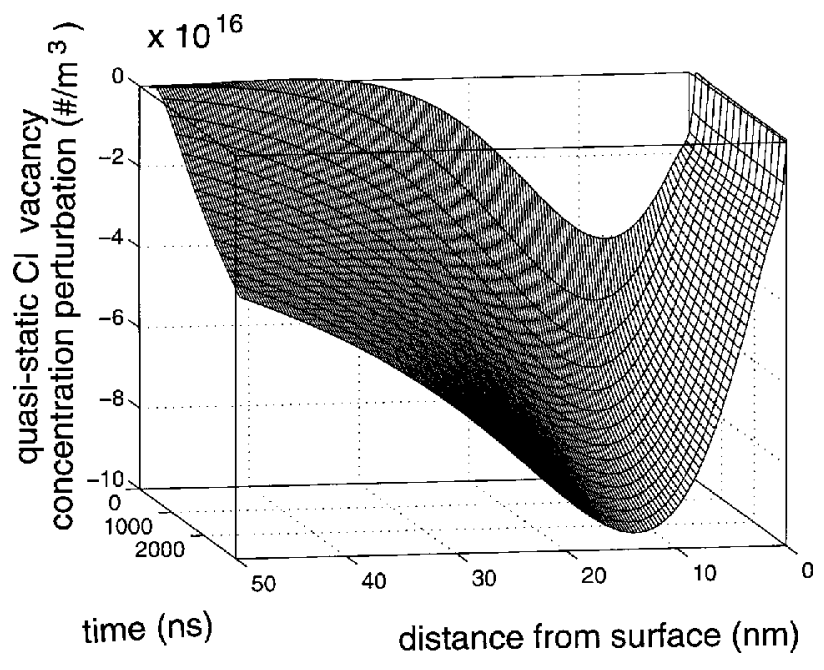

FIG. 7. The QS Cl vacancy concentration perturbations near the surface.

For the "no sink" condition (19), the QS results generally showed an electrochemical potential gradient building up to oppose the ponderomotive driving force. A quasisteady state was reached in which we observed a "double-layer" structure. There was a QS enhancement of the vacancy concentrations right near the surface, and this was balanced by a subsurface depletion region over the scale length of the DH layer. In other words, the charged defects were able to redistribute themselves to achieve a uniform electrochemical potential, exactly as in the case of boundary space-charge effects. This result was physically interesting, but certainly did not lead to mass transport. We recognized that our ID model with boundary Eq. (19) did not accurately replicate the 3D reality of a presintered compact, for example. By applying HF electric fields in the $x$ direction, we perturbed the equilibrium concentrations to a second quasiequilibrium state, but the vacancies would have wanted to diffuse down the concentration gradients in the $y$ and $z$ directions that were not included in the model. We therefore considered two ways to modify the model. Converting the model to $2 \mathrm{D}$ or 3D was one possibility, but it would have made the simulations computationally burdensome. We decided rather to change the surface boundary condition to allow for some interaction of the vacancies with the "environment." Physically, this could represent vacancy annihilation/creation at a grain boundary or free surface, or it might represent vacancies diffusing away in the $y$ and $z$ directions. We therefore believe that boundary conditions (20) or (21) were more appropriate for the types of effects we wished to study.

In Figs. 7 and 8, we plot the spatial and temporal evolution of the time-averaged concentration perturbations for $\mathrm{Cl}$ and $\mathrm{Na}$ vacancies, respectively. The "viewing angle" for these 3D plots is now slightly different than for the previous ones. The surface boundary is at the right and the bulk towards the left. The length displayed was truncated to better show the effects near the surface, although the simulation was carried out to greater depths. The time axis evolves from the back forwards. The first time-line plotted is after 200 cycles (200 ns) and the last is after 3000 cycles.

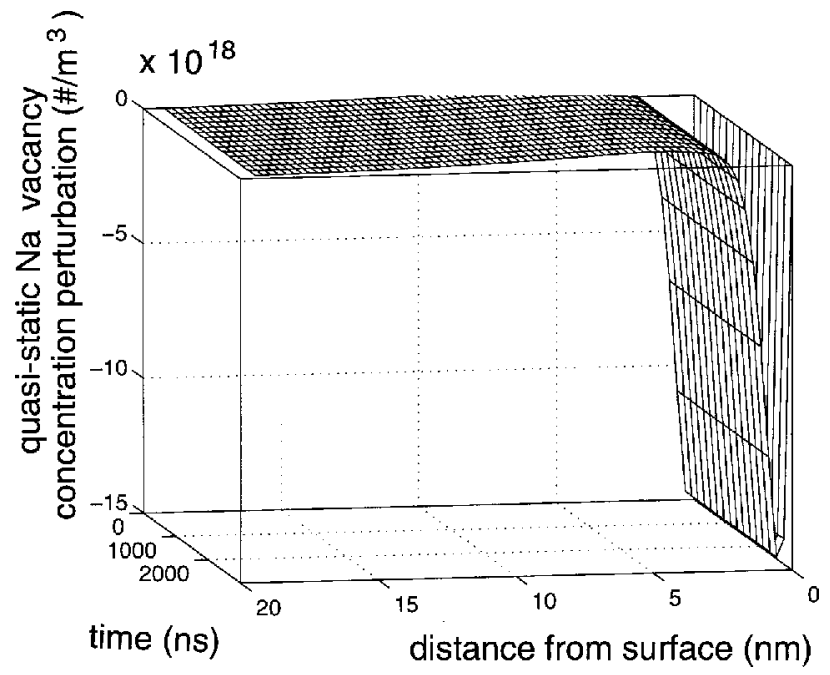

FIG. 8. The QS Na vacancy concentration perturbations near the surface.

For $\mathrm{Cl}$ vacancies, the $\mathrm{QS}$ concentration perturbations in Fig. 7 were noted to be negative except for a small positive value right at the surface. The $\mathrm{Cl}$ vacancies were slowly being depleted, and the depletion region worked inwards. This depletion surpassed the length of the DH screening distance and continued working inwards to the inner boundary of the computer model.

The behavior for Na vacancies is seen to be quite different in Fig. 8. There was a large negative "spike" within 2 $\mathrm{nm}$ of the surface, followed by a slightly positive concentration perturbation at depths beyond $2-3 \mathrm{~nm}$. This case was atypical because the $\mathrm{Cl}$ vacancies were depleted in the subsurface region, while the $\mathrm{Na}$ vacancies were enhanced. In most of the simulations, both species were depleted in the subsurface region because the ponderomotive action drove both vacancy types to the surface where they were annihilated. Since they were destroyed at the surface, the ponderomotive force continued to "pump" them to the surface, slowly depleting the subsurface region. Although such depletion did occur in this case, as is seen in Fig. 9, the positive

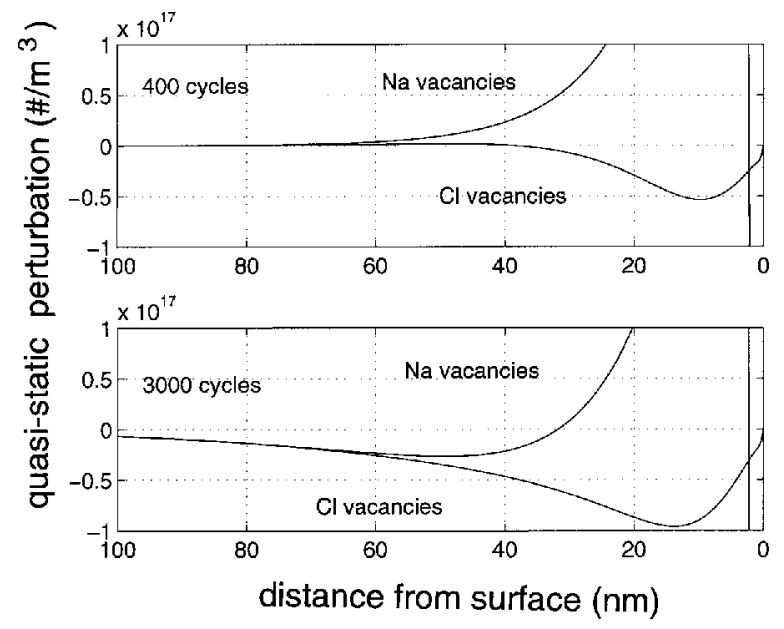

FIG. 9. The QS concentration perturbations after 400 cycles and 3000 cycles. 


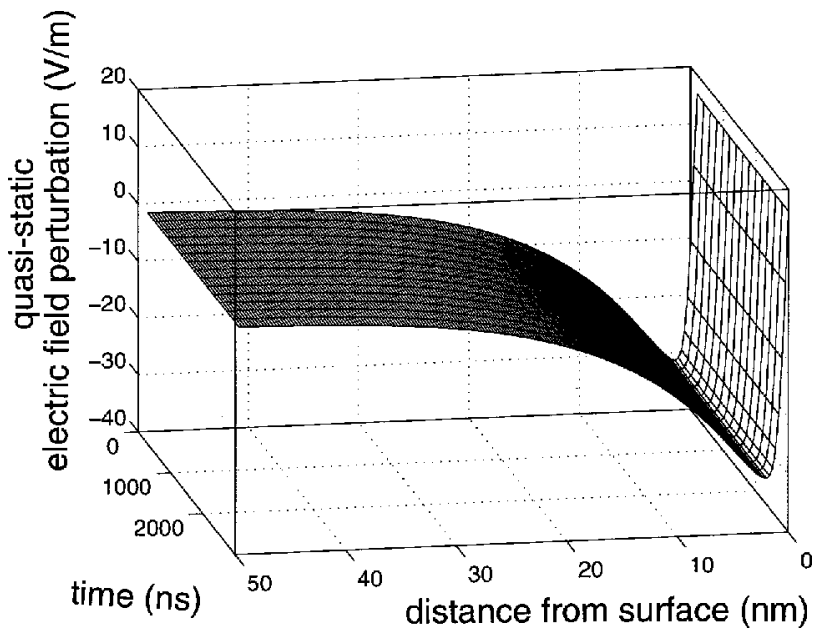

FIG. 10. The QS $E$ field perturbations near the surface.

$\mathrm{Na}$ vacancy concentration perturbation from $\sim 2$ to $20 \mathrm{~nm}$ severely throttled the depletion effect.

In Fig. 9 we plot "snapshots" of both QS concentration perturbations on a finer scale to examine more details of profiles shown in Figs. 7 and 8. The entire QS perturbation profile for $\mathrm{Cl}$ vacancies is clearly shown. After 400 cycles, there was a slightly positive region between 40 and $70 \mathrm{~nm}$ below the surface, but it was otherwise negative. Going from left (crystal interior) to right (crystal surface), the $\mathrm{Na}$ vacancy concentration perturbation started to rise at about 70 $\mathrm{nm}$ below the surface, went offscale at about $25 \mathrm{~nm}$, and came back down again at about $2-3 \mathrm{~nm}$. The almost vertical line seen at 2-3 nm corresponds to the rapid dropoff into the large negative spike seen in Fig. 8. After 3000 ns, we see the $\mathrm{Cl}$ vacancy depletion worked inwards so that the entire profile is now negative. More interestingly, the $\mathrm{Na}$ vacancy concentration was becoming depleted and, in fact, corresponded to the $\mathrm{Cl}$ vacancy concentration below $60 \mathrm{~nm}$. Because the two vacancy concentration perturbations were unequal at depths above $60 \mathrm{~nm}$, there was a space-charge field resulting from the disparity. We see in Fig. 10 the QS E-field perturbation caused by these unequal vacancy concentrations. We thus had a boundary space charge condition similar to the initial conditions. The $\mathrm{Na}$ vacancy concentration was enhanced, the $\mathrm{Cl}$ vacancy concentration was decreased, and a negative space-charge $E$ field helped to keep it all in check. The disparity in concentrations and resulting $E$ field thus acted to throttle vacancy transport to the surface.

For comparison, we now present results from another simulation run with $1000 \mathrm{ppm}$ doping, $900 \mathrm{~K}$ temperature, and a microwave field of $10^{6} \mathrm{~V} / \mathrm{m}$ at $1 \mathrm{GHz}$. The behavior of the QS results from this case were qualitatively representative of the results seen typically, but the magnitude of the QS results did vary by several orders of magnitude over the variable space probed in other simulations.

Figure 11 shows the QS concentration perturbations of $\mathrm{Cl}$ vacancies for this second case. There was a small positive value at the surface and a large subsurface depletion region that was seen to propagate inwards rather quickly. We are only plotting the $50 \mathrm{~nm}$ closest to the surface, although the inner boundary is at about $250 \mathrm{~nm}$. After 3000 cycles $(3 \mathrm{~ms})$,

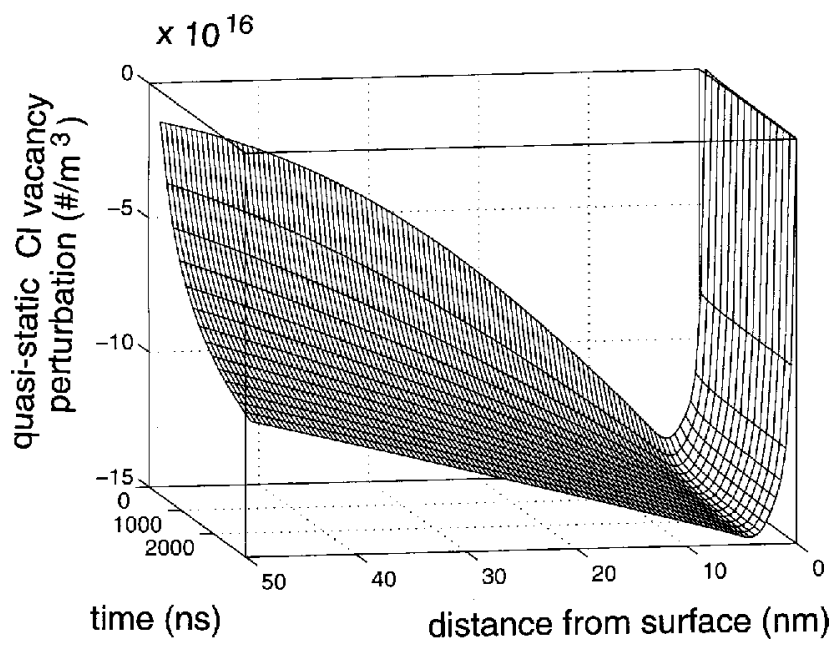

FIG. 11. The QS Cl vacancy concentration perturbations for the second case with a $\mathrm{NaCl}$ crystal at $900 \mathrm{~K}$ and 1000 ppm doping level.

the QS perturbations extended inwards almost as far as the inner boundary. As mentioned above, the bulk boundary condition did not allow for any concentration perturbations, so it was not ideally suited for studying the quasistatic problem for longer times. It was clear, though, from the behavior we see in Fig. 11 that the depletion region would continue to propagate inwards following diffusion-type behavior. These results therefore showed that the ponderomotive effect, combined with some type of surface sink condition, could drive mass transport over longer distance scales.

What distinguished this case from the previous case study was that the Na vacancy concentrations were also depleted in the subsurface regime. This is shown in Fig. 12. The large negative spike occurred within the diffusion length of the ponderomotive effect $(\sim 4-5 \mathrm{~nm})$, but its structure did not evolve with time. However. if we zoom in and examine the QS concentration snapshots on a finer scale, as we do in Fig. 13, we see that the Na vacancy concentration was evolving in unison with the $\mathrm{Cl}$ vacancy concentration. The $\mathrm{Na}$ vacancies were being depleted on the exact same scale as

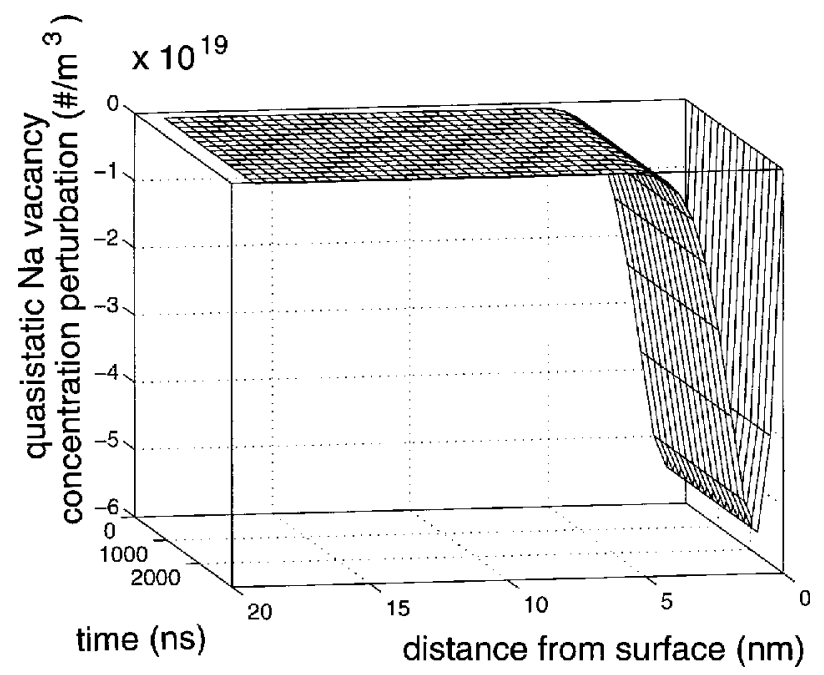

FIG. 12. The QS Na vacancy concentration perturbations for the second case with a $\mathrm{NaCl}$ crystal at $900 \mathrm{~K}$ and 1000 ppm doping level. 


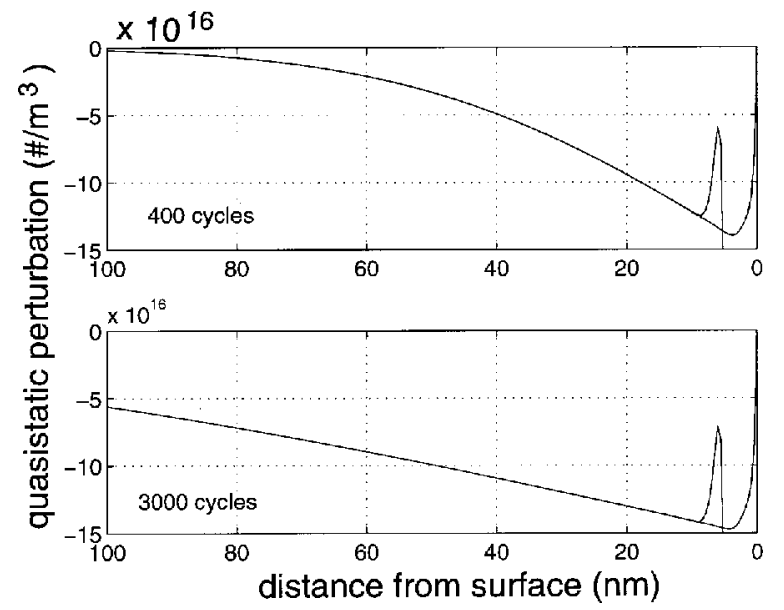

FIG. 13. The QS concentration perturbations for the second case after 400 cycles and 3000 cycles.

were the $\mathrm{Cl}$ vacancies. (The spike seen at $\sim 7 \mathrm{~nm}$ depth is in the $\mathrm{Na}$ vacancy concentration profile and occurs at the transition between near-surface ponderomotive-driven depletion and the subsurface diffusion-driven depletion.)

Because the subsurface concentrations of the two species tracked so well, this was clearly a case of charge conservation and coupled diffusion. We therefore saw no spacecharge field resulting from these perturbations. The QS $E$-field perturbation had a large value at the surface that physically corresponded to the large near-surface $\mathrm{Na}$ vacancy concentration spike seen in Fig. 12. However, in the subsurface region, the field was essentially zero. Since there was no electrical potential gradient to oppose the chemical potential gradient in this region, diffusion-type fluxes occurred and continued to transport mass over long time and distance scales.

It should be clear from the QS results presented in this section that the nature of the ponderomotive model was rather complicated even when applied to a simplified, 1D, single-crystal specimen. Other complexities abound and have not yet been fully investigated. For example, if the diffusion length outdistanced the DH length, we would expect a completely different QS response.

\section{Driving force results}

A major goal of the computer modeling was to obtain a quantitative estimate for the ponderomotive driving force ( $p m f$ ) so that we could compare it to driving forces for other mass transport processes. In this section we discuss exactly how we calculated the pmf and then compared it to other driving forces.

In the computer model, the $p m f$ was calculated from the quasistatic fluxes by dividing them into a transport coefficient $(L)$ and a driving force $(p m f)$ :

$$
J_{n}^{q s}=L_{n} p m f .
$$

From Eqs. (12) and (13), we were then able to identify:

$$
L_{n}=\left(\frac{D_{n} C_{n}^{i}}{k T}\right)
$$

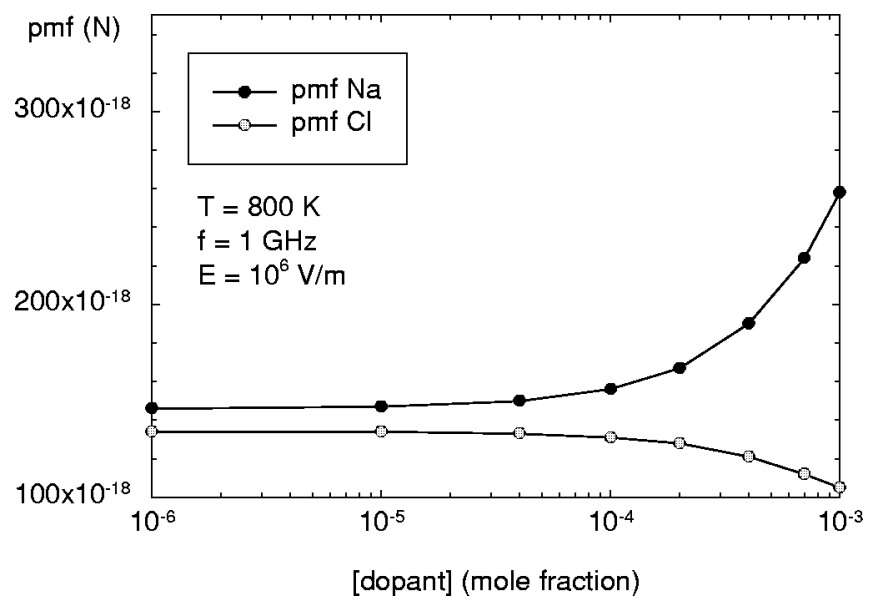

FIG. 14. The pmf characterized vs the divalent cation doping level.

and using \langle\rangle to denote time averaging over one cycle:

$$
p m f \equiv\left\langle\frac{C_{n}^{p}}{C_{n}^{i}} q_{n}\left(E^{m w}+E^{p}\right)\right\rangle .
$$

The ponderomotive driving force was thus derived only from the flux terms involving two sinusoidally varying terms multiplied by one another. Other QS transport occurred through the $\mathrm{J} 2, J 4$, and $J 6$ flux terms, but these were not driven by ponderomotive action. The $J 2$ (diffusion) term was the principal means by which vacancies were transported towards the surface, but the $J 7$ and $J 8$ (ponderomotive) terms were priming the diffusion pump by driving vacancies to the surface, where they were consumed. We therefore chose to calculate the $p m f$ from the perturbation values at the surface of the crystal. The calculation was done in units of Newtons, so that direct comparison with other driving forces was easily accomplished.

In a series of simulations, we measured the $p m f$ for both vacancy species over the variable ranges listed in Table I. The baseline case was the same as the example case in Table I except that a doping of $10^{-4}$ was used instead of $10^{-5}$. From this baseline, we then changed one variable at a time while keeping the other variables constant. We present, therefore, only a few "slices" through the four-dimensional variable space. The simulations all used boundary condition (21), but we will also present, for comparison purposes, some results obtained from the "no sink" condition (19).

The first variable of interest was the microwave field strength (in $\mathrm{V} / \mathrm{m}$ ). We found the relationship between the $p m f$ and the square of the $E$ field to be exactly linear (ranging from about $10^{-18} \mathrm{~N}$ at $10^{5} \mathrm{~V} / \mathrm{m}$ to $10^{-16} \mathrm{~N}$ at $10^{6} \mathrm{~V} / \mathrm{m}$ ). This followed the general predictions of the analytical mode $^{11}$ and both the theoretical analysis and experimental results for our ionic current measurements. ${ }^{12}$ In fact, we considered the observed linear behavior to be another successful test of the validity of the computer model. We observed in the HF results, for example, that magnitude of the flux components $J 2, J 4, J 5$, and $J 6$ all increased linearly with the $E$ field, while $J 7$ and $J 8$ increased linearly with the square of the field.

Figure 14 shows how the pmf varied as the simulated 


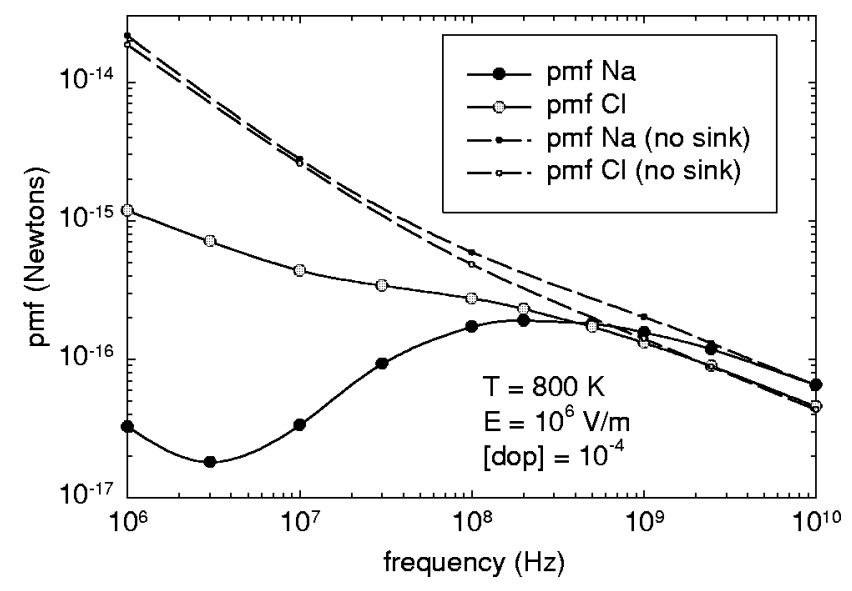

FIG. 15. The $p m f$ characterized vs the microwave frequency.

crystal was doped with divalent impurities. The $p m f$ changed by less than a factor of two, although it should be remembered that the transport coefficient [Eq. (27)] will vary much more with doping. We suspected that the $p m f$ started to change at higher doping levels because the DH length was becoming shorter, and the ponderomotive contribution from the concentration gradients became more important. At low doping levels, the ponderomotive action derived mostly from the boundary condition and was therefore unrelated to the defect concentrations.

Moving now to frequency, we characterized the $p m f$ versus a wide spectrum of high frequency excitations, as is shown in Fig. 15. The dashed lines indicate $p m f$ values calculated from simulations with the "no sink" boundary condition. In that case, the $p m f$ was seen to rise to comparatively large values at lower frequencies, because the longer time period allowed for more perturbation of the vacancy concentrations. However, as our QS results showed, this " no sink" case lead to a quasiequilibrium condition and not longrange mass transport. The pmf values resulting from the "sink" boundary condition had a more complex nature. We strongly suspect that they dropped off at lower frequencies because the HF vacancy perturbations at the surface had more time to react and be annihilated. This happened more for $\mathrm{Na}$ vacancies because of their higher mobility. It did not happen in the "no sink" condition, since defects were not allowed to be annihilated at the surface in that case. One of the more intriguing features seen in Fig. 15 for the "sink" condition is the dip in the pmf for Na vacancies right around $3 \mathrm{MHz}$. This frequency corresponded to the condition when the ionic conductivity $(\sigma)$ was equivalent to the dielectric conductivity $(\omega \varepsilon)$. Below this frequency, therefore, the diffusion length was larger than the DH length, while the opposite was true above that frequency.

Finally, we present in Fig. 16 the calculated $p m f$ values versus the crystal temperature. We again see the values going through a maximum and then dropping off. Analogous to the above argument, we believe this occurred because at higher temperatures the defects were more mobile and reacted (were annihilated) faster at the surface. The values measured from the "no sink" simulations are also plotted here for comparison, and they were identical at lower temperatures, just as

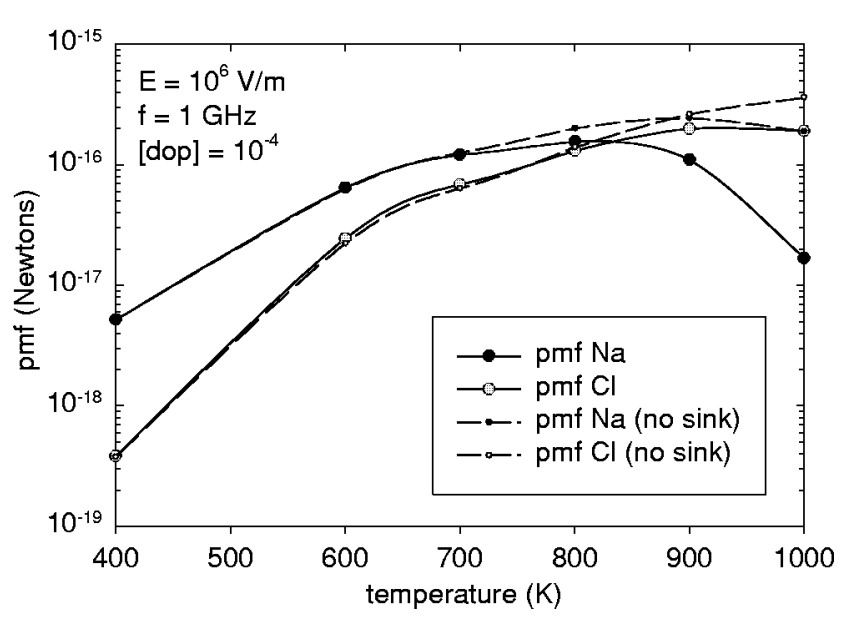

FIG. 16. The pmf characterized vs the crystal temperature.

they were identical at higher frequencies in Fig. 15. The "sink" values only started to deviate from the "no sink" values at around $700 \mathrm{~K}$.

The humps seen in Figs. 15 and 16 indicate that there are optimal values for frequency and temperature. In the four slices of variable space presented here, we have probably not happened upon the largest possible $p m f$ value, but we nonetheless have acquired a good quantitative estimate of the ponderomotive driving force. From the results shown in Figs. 14 to 16 , we can estimate that the $p m f$ falls in a range of $10^{-19}-10^{-15} \mathrm{~N}$ and more typically within $10^{-18}-10^{-16}$ $\mathrm{N}$.

In Table II we compare the microwave $p m f$ with other driving forces that we have calculated. The $p m f$ is larger than the driving force for joining and may therefore explain the rate enhancements seen during microwave joining. We find that the $p m f$ falls between the essentially zero driving force for isotope diffusion and the larger driving force for chemical diffusion. This may explain why, when using microwave heating, large enhancements of isotope diffusion were experimentally observed ${ }^{6}$ but no enhancement of chemical interdiffusion was seen ${ }^{10}$ unless diffusion occurred over long distances, ${ }^{5}$ such that the chemical driving force dropped to the equivalent level of the ponderomotive driving force.

Compared to the sintering driving force, the $p m f$ is equivalent or larger, especially compared to the intermediate

TABLE II. Comparison of microwave pmf and other thermochemical driving forces for ionic transport in $\mathrm{NaCl}$.

\begin{tabular}{lc}
\hline \hline \multicolumn{1}{c}{ Transport driving force } & Typical magnitude $(\mathrm{N})$ \\
\hline Sintering $1 \mu \mathrm{m}$ powder-initial stage & $\sim 10^{-16}$ \\
Sintering $1 \mu \mathrm{m}$ powder-intermediate & $\sim 10^{-18}$ \\
stage & $<10^{-18}$ \\
Joining two flat faces & $\sim 10^{-19}$ \\
Temperature gradients existing during & \\
microwave heating & $\sim 10^{-16}$ to $\infty$ \\
Chemical inter-diffusion (depending on & $\ll 10^{-19}$ \\
conc. gradients) & $\sim 10^{-19}-10^{-15}$ \\
Tracer isotope diffusion & \\
Microwave $p m f$ & \\
\hline \hline
\end{tabular}




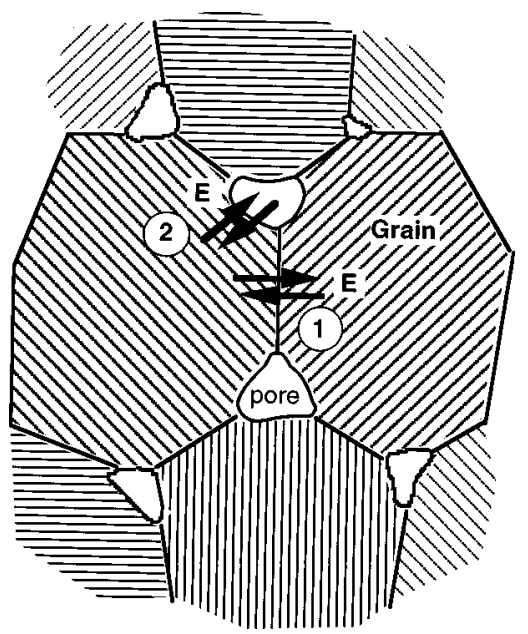

FIG. 17. Consideration of the ponderomotive effect in a porous compact.

stages of sintering. The pmf could therefore explain why more rate enhancement is observed during the intermediate stages of microwave sintering. ${ }^{2}$ Some questions still remain how the ponderomotive fluxes would impact the sintering process because of the complicated three dimensional geometries in powdered compacts. We have sketched in Fig. 17 a grain boundary between two pores in a porous compact. For case (1) when the $E$ field is oriented perpendicular to the boundary, vacancies would be driven to the boundary and depleted within a certain range of the boundary. This would then attract more vacancies from the pore into the grain and grain boundary, and the ponderomotive effect would therefore assist the densification process. However, in case (2), when the field is perpendicular to the free surface of the pore, the flux of vacancies would be in the wrong direction and would impede the sintering process. In a powdered compact, one would expect to find a completely randomized orientation of the $E$ field with respect to the grain boundary. By way of speculation, though, we would expect that the boundary conditions at the grain boundary would differ from the boundary conditions at the free surface. A true experimental test of this hypothesis might be to measure directional densification rates for a sample situated in a single mode cavity where the field orientation with respect to the sample is known and controlled. If, for example, the field is oriented along the axis of a cylindrical sample, one might see greater shrinkage in that direction compared to the radial direction. This is clearly an area that warrants further investigation.

Finally, it is instructive to speculate on the possible relevance of microwave ponderomotive forces to reports of enhanced solid state reaction rates in microwave-heated carbides, nitrides, or other ostensibly covalent (nonionic) materials. For example, the carbothermal reduction of a $\mathrm{TiO}_{2}$-and-graphite powder mixture to $\mathrm{TiC}$ and $\mathrm{CO}_{2}$ was observed $^{20}$ to occur three times faster during microwave heating as compared with conventional heating at the same temperature. Unreasonably high temperature errors of 500$600{ }^{\circ} \mathrm{C}$ (out of a typical process temperature of $1500^{\circ} \mathrm{C}$ ) would be required to explain these results solely on the basis of temperature mismeasurement arguments. An intriguing point in the context of microwave $p m f$ requires ionized at- oms to manifest its influence. One possible explanation is realized by consulting research which establish that there is a nonnegligible fractional ionicity to the interatomic bonds, as well as mobile point defects (e.g., carbon vacancies) in $\mathrm{TiC}^{21,22}$ In fact, the fractional ionization is of the order of one electron charge, which is in good agreement with predictions based on Pauling's electronegativity scale. ${ }^{23}$ Hence, there are indeed opportunities for microwave field coupling to-and, thus, microwave pmf influence on-the mobile atomic species in solids that are conventionally characterized as covalent. This also is an area worthy of further inquiry.

\section{SUMMARY AND CONCLUSIONS}

To complement the previous analytical treatment of the ponderomotive effect, we developed a numerical model with thorough consideration of boundary conditions, initial conditions, and numerical techniques. The model, based on continuum equations, can be applied to many different materials. We presented results from simulations of the ponderomotive effect in $\mathrm{NaCl}$. The static results were important for determining the length scales over which the concentration gradients and ponderomotive effects occurred. Plots of the highfrequency results were powerful visual aids for showing the vacancy flux rectification due to the initial concentration gradients and the boundary conditions. The time-averaged quasistatic results proved that the ponderomotive force could pump defects to the crystal boundary and drive long-range mass transport well into the interior of the crystal. Finally, the magnitude of the ponderomotive driving force was explored over a wide variable range, and the results explain why microwave-enhanced mass transport is observed in certain mass transport processes but not in others.

\section{ACKNOWLEDGMENTS}

The authors acknowledge the encouragement and helpful comments of many colleagues. We also appreciate John Hickland for his assistance with preparing the figures. This work was supported in part by the National Science Foundation (Grants ECS-9057675, MSM-8657164, and CTW9526035), the NASA Graduate Student Researcher Program, and the U.S. Electric Power Research Institute.

${ }^{1}$ W. H. Sutton, Am. Ceram. Soc. Bull. 68, 376 (1989).

${ }^{2}$ R. Wroe and A. T. Rowley, J. Mater. Sci. 31, 2019 (1996).

${ }^{3}$ M. A. Janney and H. D. Kimrey, in Advances in Sintering, edited by J. Bleninger and C. Handwerker (American Ceramic Society, Westerville, $\mathrm{OH}, 1990)$.

${ }^{4}$ M. A. Janney, H. D. Kimrey, M. A. Schmidt, and J. O. Kiggans, J. Am. Ceram. Soc. 74, 1675 (1991).

${ }^{5}$ Z. Fathi, D. E. Clark, and R. Hutcheon, in Microwave Processing of Materials III, edited by R. L. Beatty, W. H. Sutton, and M. F. Iskander, MRS Symp. Proc. No. 269 (Materials Research Society, Pittsburgh, 1992), p. 347.

${ }^{6}$ M. A. Janney and H. D. Kimrey, in Microwave Processing of Materials II, edited by W. B. Snyder, Jr., W. H. Sutton, M. F. Iskander, and D. L. Johnson, MRS Symp. Proc. No. 189 (Materials Research Society, Pittsburgh, 1990), p. 215.

${ }^{7}$ D. Palaith, R. Silberglitt, C. C. M. Wu, R. Kleiner, and E. Libelo, Am. Ceram. Soc. Bull. 68, 1601 (1989).

${ }^{8}$ J. G. P. Binner, P. A. Davis, T. E. Cross, and J. A. Fernie, in Microwaves: Theory and Application in Materials Processing III, edited by D. E. Clark, 
D. C. Folz, S. J. Oda, and R. Silberglitt, Ceram. Trans. No. 59 (American Ceramic Society, Westerville, OH, 1993), p. 335.

${ }^{9}$ T. N. Tiegs, K. L. Ploetz, J. O. Kiggans, and R. L. Yeckley, in Microwaves: Theory and Application in Materials Processing II, edited by D. E. Clark, W. R. Tingra, and J. R. Laia, Ceram. Trans. No. 36 (American Ceramic Society, Westerville, OH, 1993), p. 259.

${ }^{10}$ K. I. Rybakov and V. E. Semenov, Phys. Rev. B 49, 64 (1994).

${ }^{11}$ K. I. Rybakov, V. E. Semenov, S. A. Freeman, J. H. Booske, and R. F. Cooper, Phys. Rev. B 55, 3559 (1997).

${ }^{12}$ S. A. Freeman, J. H. Booske, and R. F. Cooper, Phys. Rev. Lett. 74, 2042 (1995).

${ }^{13}$ K. L. Kliewer and J. S. Koehler, Phys. Rev. 140, A1226 (1965).

${ }^{14}$ R. B. Poeppel and J. M. Blakely, Surf. Sci. 15, 507 (1969).

${ }^{15}$ I. E. Hooton and P. W. M. Jacobs, J. Phys. Chem. Solids 51, 1207 (1990).
${ }^{16}$ K. M. Kramer and W. N. G. Hitchon, Comput. Phys. Commun. 85, 167 (1995).

${ }^{17}$ K. M. Kramer and W. N. G. Hitchon, Comput. Phys. Commun. 93, 1 (1996).

${ }^{18}$ S. Selberherr, Analysis and Simulation of Semiconductor Devices (Springer, Vienna, 1984).

${ }^{19}$ D. E. Potter, Computational Physics (Wiley, New York, 1973).

${ }^{20}$ J. G. P. Binner, N. A. Hassine, and T. E. Cross, J. Mater. Sci. 30, 5389 (1995).

${ }^{21}$ W. Schmickler and J. W. Schultze, Ber. Bunsenges. Phys. Chem. 96, 760 (1992).

${ }^{22}$ A. L. Ivanovskii, D. L. Novikov, V. A. Gubanov, and G. P. Shveikin, Phys. Chem. Mech. Surf. 8, 349 (1992).

${ }^{23}$ L. Pauling, The Nature of the Chemical Bond, 3rd ed. (Cornell University Press, Ithaca, NY, 1960), pp. 88-102. 